\title{
Multi-antenna techniques for interference mitigation in GNSS signal acquisition
}

\author{
Javier Arribas* , Carles Fernández-Prades and Pau Closas
}

\begin{abstract}
This paper addresses the signal acquisition problem in the presence of interferences using antenna arrays in the general framework of Global Navigation Satellite Systems receivers. We describe and compare two different approaches: the first one is based on the generalized likelihood ratio test (GLRT) detector directly applied on the array snapshots, and the second one uses a digital beamformer as a spatial filter to mitigate the interferences and acquire the signal using the beamformer output. We show that the array-based GLRT acquisition is equivalent to a conventional acquisition based on the output of a time reference beamformer. The test statistics of the techniques are analyzed in terms of the probability of detection and false alarm. Monte Carlo simulations using the Galileo E1 signal structure support the theoretical results, even when the array is moderately uncalibrated.
\end{abstract}

\section{Introduction}

The fact that current and planned accurate positioning and timing services rely on Global Navigation Satellite Systems (GNSS) has raised the concern about possible denial of service situations, as reported in [1] among others. This is specially important when GNSS are used in safety-critical or mission-critical operations, such as their integration into civil aviation or in the synchronization of power distribution networks [2].

Examining the threats against the GNSS service quality and availability, radio frequency interferences (RFI) are considered to be potentially dangerous threats to GNSS applications, in particular where a risk on human life may occur. The proliferation of portable jamming devices [3], the evolution of spoofing or meaconing techniques, and the collateral interference effects caused by a possible deployment of a 4G Long Term Evolution network in the $1,552.7-\mathrm{MHz}$ band [4] drive to the inclusion of countermeasures against RFI.

GNSS receivers can make use of time and frequency diversity to mitigate interferences, although the performance of these techniques is compromised in low signal-to-noise ratio (SNR) scenarios or in the presence of wideband RFI. Complementarily, antenna array-based

\footnotetext{
*Correspondence: jarribas@cttc.cat

Centre Tecnològic de Telecomunicacions de Catalunya (CTTC), Parc Mediterrani de la Tecnologia, Av. Carl Friedrich Gauss 7, Castelldefels,
} Barcelona 08860, Spain receivers can benefit from spatial domain processing and thus mitigate the interferences exploiting spatial diversity. In GNSS receivers, antenna arrays are usually applied to signal tracking, where the algorithms may have access to an initial coarse estimation of the signal synchronization parameters and the array attitude (and thus an estimation of the direction of arrival of the desired signals). Hence, tracking depends on signal acquisition, which constitutes a performance bottleneck in the presence of RFI.

In this paper we address the signal acquisition problem for antenna array-based receivers using two different approaches. The first one was recently presented by the authors in [5]. It is based on the generalized likelihood ratio test (GLRT) detector operating directly with the array snapshots. The second approach uses a digital beamformer as a spatial filter to mitigate the interferences and then detect the signal using the beamformer output. We considered three different designs: the time reference beamformer (TRB), which relies on a priori knowledge of a reference waveform; the minimum variance distortionless response (MVDR), which relies on a priori knowledge of the spatial signature of the signal using direction-ofarrival (DOA) estimation; and the blind null-steering, which is also known as power minimization beamformer in the GNSS literature.

We prove that the proposed multi-antenna GLRT acquisition algorithm is equivalent to a TRB beamformer followed by a single-antenna acquisition. Since

\section{Springer}

(c) 2013 Arribas et al: licensee Springer. This is an Open Access article distributed under the terms of the Creative Commons Attribution License (http://creativecommons.org/licenses/by/2.0), which permits unrestricted use, distribution, and reproduction in any medium, provided the original work is properly cited. 
both approaches theoretically attain the same performance, the choice between the statistical detector and the beamformer-based acquisition is driven by implementation aspects, such as the computational complexity or the integration of the required signal processing structures into the downstream signal tracking architecture.

We analyze the detection performance when such approaches are applied, obtaining closed-form expressions in terms of detection and false alarm probabilities. The single-dwell acquisition performance under strong RFI was compared taking into account realistic scenarios where the array is moderately uncalibrated. Monte Carlo (MC) simulations for the Galileo E1 signal acquisition support the theoretical results.

The paper is organized as follows: Section 2 presents the antenna array signal model and the statistical hypotheses. Section 3 briefly describes the GLRT algorithm, as well as the acquisition test statistic and its theoretical performance. In Section 4 we present the acquisition after the beamforming approach and we obtain its performance expressions. Section 5 compares the algorithms by MC simulations, Section 6 is devoted to analyze the computational complexity associated to the selected algorithms, and finally, Section 7 concludes the paper.

\section{Signal model}

We consider the discrete baseband signal received from a single GNSS satellite with an unstructured $N$-element antenna array:

$$
\mathbf{X}(t)=\mathbf{h d}\left(t, f_{d}, \tau\right)+\mathbf{N}(t)
$$

where $\mathbf{X}(t)=\left[\mathbf{x}\left(t-(K-1) T_{s}\right) \ldots \mathbf{x}(t)\right] \in \mathbb{C}^{N \times K}$ is the space-time data matrix, with $\mathbf{x}(t)=\left[x_{1}(t) \ldots x_{N}(t)\right]^{T}$ defined as the array baseband snapshot, each row corresponding to one antenna, and $K$ is the number of captured snapshots. The acquisition time is defined as $T_{\mathrm{acq}}=K T_{s}$, where $F_{s}=1 / T_{s}$ is the sampling frequency. Vector $\mathbf{h}=$ $\left[h_{1} \ldots h_{N}\right]^{T} \in \mathbb{C}^{N \times 1}$ is a non-structured vector which includes the signal power and both the channel model and the array response. It assumes the role of the spatial signature, which is considered constant during $T_{\text {acq }}$. This channel vector not only is parametrized by the signal DOA and the antenna locations, but also includes other unmodeled phenomena like the front-end amplitude/phase responses and the electromagnetic mutual coupling between array elements and front-end channels. $\mathbf{d}\left(t, f_{d}, \tau\right)=\left[s\left(t-(K-1) T_{s}-\tau\right) e^{j 2 \pi f_{d}\left(t-(K-1) T_{s}\right)} \ldots\right.$ $\left.s(t-\tau) e^{j 2 \pi f_{d} t}\right] \in \mathbb{C}^{1 \times K}$ is a GNSS baseband direct sequence spread spectrum (DSSS) signal with normalized power and known structure $s(t)$, received with a propagation delay $\tau$ and a Doppler frequency $f_{d}$, considered constant during $T_{\text {acq. }}$. The general DSSS signal structure can be written as $s(t)=\sum_{k=-\infty}^{\infty} c_{k} g_{k}\left(t-k T_{c}\right)$, where $c_{k}$ is the spreading code sequence, $g_{k}$ is the receiving pulse, and $T_{c}$ is the code chip period of a pseudorandom noise (PRN) sequence with period $T_{\text {code }}$. This signal model can be particularized to describe a Galileo E1 signal [6], among other GNSS signals. Finally, $\mathbf{N}(t)=\left[\mathbf{n}\left(t-(K-1) T_{s}\right) \ldots \mathbf{n}(t)\right] \epsilon$ $\mathbb{C}^{N \times K}$ is a complex, circularly symmetric Gaussian vector process with a zero mean, temporally white, and spatially colored which is modeled with an arbitrary (also unknown) spatial covariance matrix $\mathbf{Q} \in \mathbb{C}^{N \times N}$ defined as $\mathrm{E}\left\{\mathbf{n}\left(t_{n}\right) \mathbf{n}^{H}\left(t_{m}\right)\right\}=\sigma^{2} \mathbf{I}+\boldsymbol{\Sigma}=\mathbf{Q} \delta_{t_{n}, t_{m}}$, where $\mathrm{E}\{\cdot\}$ stands for the expectation operator. The noise is assumed with double-sided spectral density $\sigma^{2}=\frac{N_{0}}{2} \mathrm{~W} / \mathrm{Hz}$, I is the identity matrix, $\boldsymbol{\Sigma}$ models the covariance matrix of interferences, and $\delta_{t_{n}, t_{m}}$ stands for the Kronecker delta.

For notation convenience, in the rest of the document, we group the unknown signal parameters in a vector $\boldsymbol{\theta}=\left[\mathbf{h}^{T}, f_{d}, \tau\right]^{T}$. We focus on a single satellite's signal, thus neglecting the contribution of the rest of the satellites. This assumption is realistic, considering that GNSS use pseudorandom noise codes with a high processing gain and relatively small cross-correlation among satellite codes. Therefore, the influence of other satellites can be considered as Gaussian noise and included in the thermal noise term since those signals are well below the noise floor [7].

The acquisition process determines the presence or the absence of a specific satellite signal and provides a coarse estimate of the signal synchronization parameters in order to initialize the tracking process. We define two possible hypotheses, referred to as $\mathcal{H}_{0}$ or the null hypothesis, when the searched satellite signal is absent, and $\mathcal{H}_{1}$ or the alternative hypothesis, when the signal is present. The hypotheses are defined as follows:

$$
\begin{aligned}
& \mathcal{H}_{1}: \mathbf{X}(t)=\mathbf{h d}\left(t, f_{d}, \tau\right)+\mathbf{N}(t) \text { and } \\
& \mathcal{H}_{0}: \mathbf{X}(t)=\mathbf{N}(t)
\end{aligned}
$$

The mutual exclusivity of the hypotheses forces $\|\mathbf{h}\|>0$ in $\mathcal{H}_{1}$. In the sequel, two different approaches to solve the problem are presented and compared.

\section{Signal acquisition using the GLRT detector}

The GLRT takes into account the probability density function (PDF) of $\mathbf{X}(t)$ to obtain a test function which is able to detect GNSS signals by maximizing the probability of detection $\left(P_{\mathrm{d}}\right)$ subject to a given false alarm probability $\left(P_{\mathrm{fa}}\right)$ [8]. Figure 1 shows a high-level block diagram of the proposed acquisition architecture.

The algorithm, briefly presented here, was derived and analyzed by the authors in [5]. The likelihood ratio test 


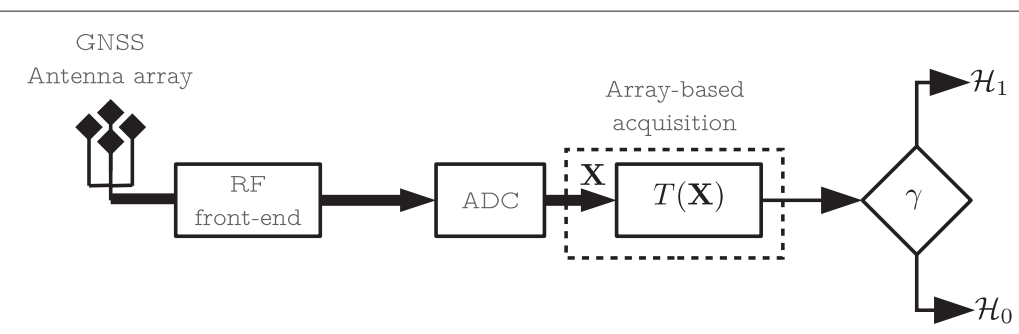

Figure 1 GLRT array-based acquisition.

statistic for testing $\mathcal{H}_{0}: \boldsymbol{\theta} \in \Theta_{0}$ versus $\mathcal{H}_{1}: \boldsymbol{\theta} \in \Theta_{0}^{c}$ (see, e.g., $[9$, p. 375]) can be expressed as

$$
T_{\mathrm{GL}}(\mathbf{X})=\frac{\sup _{0} L(\boldsymbol{\theta} ; \mathbf{X})}{\sup _{\Theta} L(\boldsymbol{\theta} ; \mathbf{X})} \leq \gamma^{\prime},
$$

where $L(\boldsymbol{\theta} ; \mathbf{X})$ stands for the likelihood function of $\boldsymbol{\theta}$ given $\mathbf{X}$, the space associated with $\Theta_{0}=\mathbf{0} \times\left[f_{d, \min }, f_{d, \max }\right] \times\left[0, T_{\text {code }}\right], \Theta_{0}^{c}$ is the complementary mutually exclusive space (i.e., the satellite signal is present $\|\mathbf{h}\|>0$ ) and $\Theta=\Theta_{0} \cup \Theta_{0}^{c}$ represents the entire parameter space. We assumed a Doppler shift $f_{d}(t)$ in the range $\left[f_{d, \min }, f_{d, \text { max }}\right]$ and a spreading code of duration $T_{\text {code }}$. The test rejects $\mathcal{H}_{0}$ if $T_{\mathrm{GL}}(\mathbf{X}) \leq \gamma^{\prime}$, where $\gamma^{\prime}$ is the detection threshold. Maximization over both the parameter space $\Theta$ and a subset of the parameter space $\Theta_{0}$ is related to the maximum likelihood estimator (MLE) of $\boldsymbol{\theta}$ (known as unrestricted $\hat{\boldsymbol{\theta}}$ and restricted $\hat{\boldsymbol{\theta}}_{0}$ estimators) [9]. Then, it is possible to write

$$
T_{\mathrm{GL}}(\mathbf{X})=\frac{L\left(\hat{\boldsymbol{\theta}}_{0} ; \mathbf{X}\right)}{L(\hat{\boldsymbol{\theta}} ; \mathbf{X})} \leq \gamma^{\prime} .
$$

Then, inserting the MLE expressions in (5), the test statistic results in (see [5] for a detailed derivation)

$$
T_{\mathrm{GL}}(\mathbf{X})=\max _{f_{d}, \tau}\left\{\hat{\mathbf{r}}_{\mathbf{x d}}^{H}\left(f_{d}, \tau\right) \hat{R}_{\mathbf{d d}}^{-1} \hat{\mathbf{R}}_{\mathbf{x x}}^{-1} \hat{\mathbf{r}}_{\mathbf{x d}}\left(f_{d}, \tau\right)\right\} \geq \gamma,
$$

where $\hat{\mathbf{R}}_{\mathbf{x x}}=\frac{1}{K} \mathbf{X} \mathbf{X}^{H}$ is an estimation of the spatial autocorrelation matrix of the array snapshots, $\hat{\mathbf{r}}_{\mathbf{x d}}=\frac{1}{K} \mathbf{X \mathbf { d } ^ { H }}$ is an estimation of the cross-correlation vector between the received array snapshots and a local satellite signal replica, and $\hat{R}_{\mathbf{d d}}=\frac{1}{K} \mathbf{d d} \mathbf{d}^{H}$ is an estimation of the satellite signal autocorrelation. We consider that $\mathbf{d}$ has normalized power $\left(\hat{R}_{\mathbf{d d}} \simeq 1\right)$. The maximization operation is inherited from the MLE expressions (see [5]). Equation (6) can be solved by an exhaustive grid search in the entire $\left(f_{d}, \tau\right)$ parameter space, and thus, grid search strategies used in the conventional single-antenna acquisition [10] can be applied ${ }^{\mathrm{a}}$. The final test statistic rejects $\mathcal{H}_{0}$ if $T_{\mathrm{GL}}(\mathbf{X}) \geq \gamma$.

The theoretical performance of the detector can be obtained assuming that $\hat{\mathbf{r}}_{\mathbf{x d}} \sim \mathcal{C N}\left(\hat{R}_{\mathbf{d d}} \mathbf{h}, \frac{\mathbf{R}_{\mathbf{x x}}}{K}\right)$ if $\mathbf{d}$ is uncorrelated with $\mathbf{N}$ and considering $\hat{\mathbf{R}}_{\mathbf{x x}} \simeq \mathbf{R}_{\mathbf{x x}}$. Then, (6) can be modeled as a quadratic form $\mathbf{z}^{H} \boldsymbol{\Sigma}^{-1} \mathbf{z}$ of a complex
Gaussian random variable, which is distributed as a noncentral chi-square $\chi_{2 N}^{2}(\delta)$ with $2 N$ degrees of freedom and $\delta=\boldsymbol{\mu}^{H} \boldsymbol{\Sigma}^{-1} \boldsymbol{\mu}$, when $\mathbf{z} \in \mathbb{C}^{N \times 1}$ and $\mathbf{z} \sim \mathcal{C N}(\boldsymbol{\mu}, \boldsymbol{\Sigma})$. Notice that the presence of $\Sigma^{-1}$ acts as a whitening transformation, and thus, the resulting distribution is independent of the structure of $\Sigma$, see, e.g., [11, p.26]. The $\chi^{2}$ noncentrality parameter can be expressed as

$$
\delta_{T_{\mathrm{GL}}}\left(\hat{\mathbf{R}}_{\mathbf{x x}}, \mathbf{h}\right)=\hat{R}_{\mathbf{d d}} \mathbf{h}^{H} \hat{\mathbf{R}}_{\mathbf{x x}}^{-1} \mathbf{h} \simeq \mathbf{h}^{H} \mathbf{R}_{\mathbf{x x}}^{-1} \mathbf{h} .
$$

The distributions of the test functions for both hypotheses are

$$
T_{\mathrm{GL}}(\mathbf{X}) \sim \begin{cases}\chi_{2 N}^{2}\left(\delta_{T_{\mathrm{GL}} ; \mathcal{H}_{1}}\right), & \text { in } \mathcal{H}_{1} \\ \chi_{2 N}^{2}\left(\delta_{T_{\mathrm{GL}} ; \mathcal{H}_{0}}\right), & \text { in } \mathcal{H}_{0} .\end{cases}
$$

Notice that $\delta_{T_{\mathrm{GL}} ; \mathcal{H}_{0}}=0$ because the satellite signal is not present $(\mathbf{h}=\mathbf{0})$. Closed-form performance expressions can be written as $P_{\mathrm{fa}}(\gamma)=1-P_{\mathcal{H}_{0}}\left(T\left(\mathbf{X} ; \mathcal{H}_{0}\right) \leq \gamma\right)$ and $P_{\mathrm{d}}(\gamma)=1-P_{\mathcal{H}_{1}}\left(T\left(\mathbf{X} ; \mathcal{H}_{1}\right) \leq \gamma\right)$, where $P_{\mathcal{H}_{0}}(\cdot)$ and $P_{\mathcal{H}_{1}}(\cdot)$ are the central and non-central $\chi_{2 N}^{2}$ cumulative density functions (CDFs), respectively:

$$
\begin{aligned}
& P_{\mathrm{fa}}(\gamma)=\exp \left\{\frac{-\gamma}{2 \sigma_{T_{\mathrm{GL}}}^{2}}\right\} \sum_{k=0}^{N-1} \frac{1}{k !}\left(\frac{\gamma}{2 \sigma_{T_{\mathrm{GL}}}^{2}}\right)^{k}, \\
& P_{\mathrm{d}}(\gamma)=\mathrm{Q}_{N}\left(\frac{\left.\sqrt{\delta_{T_{\mathrm{GL}}\left(\hat{\mathbf{R}}_{\mathbf{x x}}, \mathbf{h}\right)}}, \frac{\sqrt{\gamma}}{\sigma_{T_{\mathrm{GL}}}}\right),}{\sigma_{T_{\mathrm{GL}}}}\right)
\end{aligned}
$$

where $\sigma_{T_{\mathrm{GL}}}^{2}=\frac{1}{2 K}[5]$ and $\mathrm{Q}_{N}$ is the generalized Marcum Q-function [11] of order $N$.

\section{Signal acquisition after a beamformer processor} Another approach consists of using a beamforming stage prior to the acquisition algorithm. The beamformer output can be written as

$$
\mathbf{y}=\mathbf{w}^{H} \mathbf{X}
$$

where $\mathbf{w} \in \mathbb{C}^{N \times 1}$ is the beamweight vector. By developing the GLRT detector for a generic beamformer output in (11), the acquisition test statistic results in

$$
T_{\mathrm{DBF}}(\mathbf{y})=\max _{f_{d}, \tau}\left\{\frac{\hat{R}_{\mathbf{y d}}^{H} \hat{R}_{\mathbf{y d}}}{\hat{R}_{\mathbf{y y}}}\right\}>\gamma,
$$


where $\hat{R}_{\mathbf{y d}}=\mathbf{w}^{H} \hat{\mathbf{r}}_{\mathbf{x d}\left(\mathbf{f}_{\mathbf{d}}, \tau\right)}$ and $\hat{R}_{\mathbf{y y}}=\mathbf{w}^{H} \hat{\mathbf{R}}_{\mathbf{x x}} \mathbf{w}$. The maximization of (12) can be solved by an exhaustive grid search in the entire $\left(f_{d}, \tau\right)$ parameter space. The reader is referred to [12, p.79] for a detailed description of the algorithm. Figure 2 shows a simplified block diagram of the approach.

\subsection{Time reference beamformer}

Now we particularize the beamweight vector with the TRB, which relies on a priori knowledge of a reference waveform to minimize the mean square error between the beamformer output and a reference signal [13, p. 90]. This design criterion can be written as

$$
\hat{\mathbf{w}}_{\mathrm{TRB}}=\min _{\mathbf{w}}\left\{\left\|\mathbf{d}-\mathbf{w}^{H} \mathbf{X}\right\|^{2}\right\},
$$

where $\|\cdot\|$ stands for the $l^{2}$-norm of a vector. The closedform solution for the optimum weightsis

$$
\hat{\mathbf{w}}_{\mathrm{TRB}}=\hat{\mathbf{R}}_{\mathbf{x x}}^{-1} \hat{\mathbf{r}}_{\mathbf{x d}} \text {. }
$$

By inserting (14) in (12), and using $\hat{R}_{\text {yy }}$ and $\hat{R}_{\text {yd }}$ definitions, $T_{\mathrm{DBF}}(\mathbf{y})$ can be rewritten after some mathematical manipulation as

$$
T_{\mathrm{TRB}}(\mathbf{y})=\frac{\left(\hat{\mathbf{w}}_{\mathrm{TRB}}^{H} \hat{\mathbf{r}}_{\mathbf{x d}}\right)^{H} \hat{\mathbf{w}}_{\mathrm{TRB}}^{H} \hat{\mathbf{r}}_{\mathbf{x d}}}{\hat{\mathbf{w}}_{\mathrm{TRB}}^{H} \hat{\mathbf{R}}_{\mathbf{x x}} \hat{\mathbf{w}}_{\mathrm{TRB}}}=\hat{\mathbf{r}}_{\mathbf{x d}}^{H} \hat{\mathbf{R}}_{\mathbf{x x}}^{-1} \hat{\mathbf{r}}_{\mathbf{x d}} .
$$

As a result, the test function obtained using the conventional acquisition after the TRB beamformer algorithm is exactly the same as the test function obtained by the GLRT detector. Consequently, both algorithms become equivalent and share its optimality in terms of maximizing the SNR [13]. In the acquisition search grid, each of the bins has a particular set of beamweights associated to the local reference signal. The beamforming structure in the acquisition provides some benefits since it can be reused by a tracking algorithm afterward. Since the test statistic is the same as in the array-based GLRT, the performance of the approach can be modeled using the theoretical detection and false alarm probabilities shown in (10) and (9), respectively. The theoretical PDF was validated by $\mathrm{MC}$ simulations for a Galileo E1 signal acquisition in the absence of interferences, as shown in Figure 3.

\subsection{MVDR beamformer with known signal DOA}

On the other hand, the MVDR beamformer [13] relies on an estimation of the signal DOA. The design equation can be written as

$$
\begin{aligned}
& \hat{\mathbf{w}}_{\mathrm{MVDR}}=\min _{\mathbf{w}}\left\{\mathbf{w}^{H} \mathbf{R}_{\mathbf{x x}} \mathbf{w}\right\} \\
& \text { s.t. } \mathbf{w}^{H} \mathbf{h}_{0}=1,
\end{aligned}
$$

where the steering vector $\mathbf{h}_{0}$ points to the desired signal DOA. This problem has the optimal beamweight vector:

$$
\hat{\mathbf{w}}_{\mathrm{MVDR}}=\frac{\hat{\mathbf{R}}_{\mathbf{x x}}^{-1} \mathbf{h}_{0}}{\mathbf{h}_{0}^{H} \hat{\mathbf{R}}_{\mathbf{x x}}^{-1} \mathbf{h}_{0}} .
$$

$T_{\mathrm{DBF}}(\mathbf{y})$ can be rewritten by inserting (17) in $\hat{R}_{\mathbf{y y}}$ and $\hat{R}_{\text {yd }}$. After a straightforward manipulation of (12), the test statistic is obtained as

$T_{\mathrm{MVDR}}(\mathbf{y})=\frac{\hat{\mathbf{r}}_{\mathbf{x d}}^{H}\left(\hat{\mathbf{R}}_{\mathbf{x x}}^{-1} \mathbf{h}_{0}\right)\left(\hat{\mathbf{R}}_{\mathbf{x x}}^{-1} \mathbf{h}_{0}\right)^{H} \hat{\mathbf{r}}_{\mathbf{x d}}}{\mathbf{h}_{0}^{H} \hat{\mathbf{R}}_{\mathbf{x x}}^{-1} \mathbf{h}_{0}} \simeq \frac{1}{\sigma^{2}} \frac{\left|\hat{\mathbf{r}}_{\mathbf{x d}}^{H} \mathbf{P}_{\mathbf{H}_{\mathrm{i}}}^{\perp} \mathbf{h}_{0}\right|^{2}}{\left\|\mathbf{h}_{0}^{H} \mathbf{P}_{\mathbf{H}_{\mathrm{i}}}^{\perp}\right\|^{2}}$.

$T_{\mathrm{MVDR}}(\mathbf{y})$ can be interpreted in terms of the geometric projection over the interference subspace $\mathbf{H}_{\mathrm{i}}=$ $\left[\mathbf{h}_{\mathrm{i}, 1}, \ldots, \mathbf{h}_{\mathrm{i}, M}\right] \in \mathbb{C}^{N \times M}$, where $\mathbf{h}_{\mathbf{i}, n}$ is the steering vector of the $n$th interference. If the interferences dominate the array covariance matrix, expressed as $\hat{\mathbf{R}}_{\mathbf{x x}}^{-1} \simeq \sigma^{-2} \mathbf{P}_{\mathbf{H}_{\mathrm{i}}}^{\perp}$, where $\mathbf{P}_{\mathbf{H}_{\mathrm{i}}}^{\perp}$ is the projection matrix to the subspace orthogonal to the interference subspace, then the latter expression in (18) arises. This algorithm was implemented by the German Aerospace Center (DLR) in a Galileo Safety of Life receiver prototype [14]. The DOA estimation was solved by extending the acquisition search grid to include azimuth and elevation parameters.

The theoretical performance for the acquisition after the MVDR beamformer can be modeled assuming that $\hat{R}_{\text {yd }}$ is distributed as a complex Gaussian variable $\hat{R}_{\text {yd }} \sim$ $\mathcal{C N}\left(\mu_{\hat{R}_{\mathrm{yd}}}, \sigma_{\hat{R}_{\mathrm{yd}}}^{2}\right)$, with

$$
\mu_{\hat{R}_{\mathbf{y d}}}=\mathrm{E}\left\{\hat{R}_{\mathbf{y d}}\right\}=\mathbf{w}_{\mathrm{MVDR}}^{H} \mathrm{E}\left\{\hat{\mathbf{r}}_{\mathbf{x d}}\right\}=\mathbf{w}_{\mathrm{MVDR}}^{H} R_{\mathbf{d d}} \mathbf{h}
$$

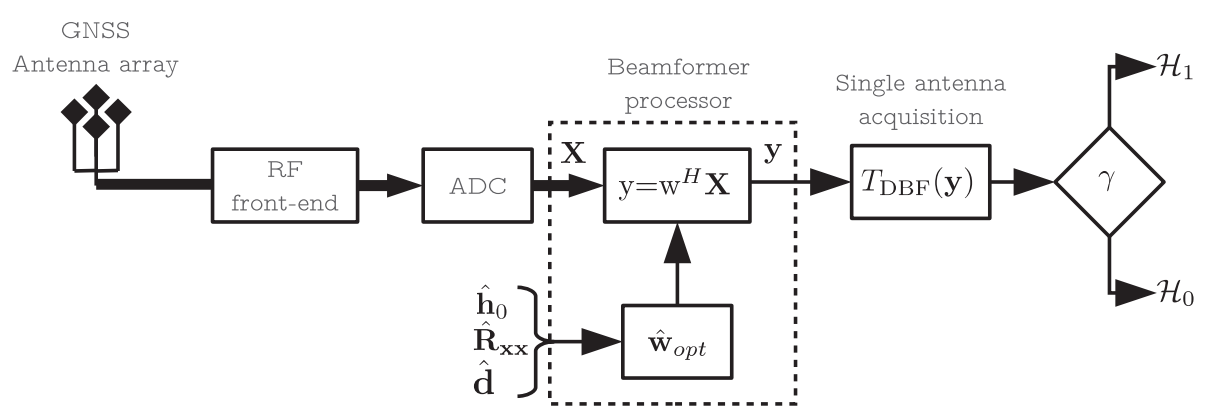

Figure 2 Acquisition after beamforming. 


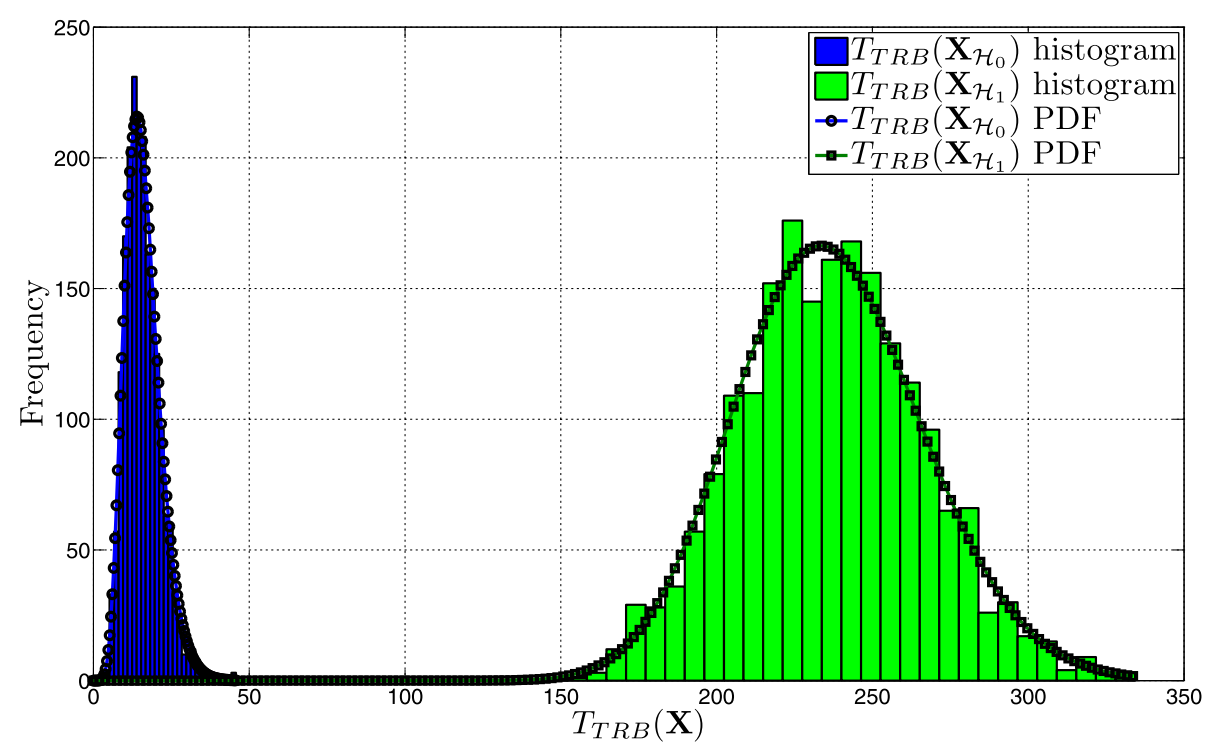

Figure $3 T_{\mathrm{TRB}}(\mathrm{X})$ test statistic histograms and theoretical PDFs for 4,000 realizations and $\mathrm{CNO}=34 \mathrm{~dB}-\mathrm{Hz}$ without interferences.

and

$$
\begin{aligned}
\sigma_{\hat{R}_{\mathbf{y d}}}^{2} & =\mathrm{E}\left\{\left(\hat{R}_{\mathbf{y d}}-\mathbf{w}_{\mathrm{MVDR}}^{H} \hat{R}_{\mathbf{d d}} \mathbf{h}\right)\left(\hat{R}_{\mathbf{y d}}-\mathbf{w}_{\mathrm{MVDR}}^{H} \hat{R}_{\mathbf{d d}} \mathbf{h}\right)^{H}\right\}= \\
& =\mathbf{w}_{\mathrm{MVDR}}^{H} \frac{\mathbf{R}_{\mathbf{n n}}}{K} \mathbf{w}_{\mathrm{MVDR}} \simeq \mathbf{w}_{\mathrm{MVDR}}^{H} \frac{\mathbf{R}_{\mathbf{x x}}}{K} \mathbf{w}_{\mathrm{MVDR}}, \quad(20)
\end{aligned}
$$

where $\mathbf{R}_{\mathbf{n n}}=\mathrm{E}\left\{\frac{1}{K} \mathbf{N} \mathbf{N}^{H}\right\}$, and we considered that the beamweight vector is deterministic by assuming that $\hat{\mathbf{R}}_{\mathbf{x x}} \simeq \mathbf{R}_{\mathbf{x x}}$. The approximation in (20) is valid only for low signal power values, which is the case in GNSS [12]. In this case, $T_{\text {MVDR }}(y)$ can be analyzed as a quadratic form of $\hat{R}_{\text {yd }}$, and thus, it is distributed as a chi-square random variable $\chi_{2}^{2}\left(\delta_{T_{\mathrm{MVDR}}}\right)$ for each hypothesis:

$$
T_{\mathrm{MVDR}}(\mathbf{y}) \sim \begin{cases}\chi_{2}^{2}\left(\delta_{T_{\mathrm{MVDR}} ; \mathcal{H}_{1}}\right), & \text { in } \mathcal{H}_{1}, \\ \chi_{2}^{2}\left(\delta_{T_{\mathrm{MVDR}} ; \mathcal{H}_{0}}=0\right), & \text { in } \mathcal{H}_{0},\end{cases}
$$

where the non-centrality parameter can be written as

$$
\delta_{T_{\mathrm{MVDR}} ; \mathcal{H}_{1}}=\frac{\left|\mu_{\hat{R}_{\mathbf{y d}}}\right|^{2}}{\hat{R}_{\mathbf{y y}}}=\frac{\mathbf{w}_{\mathrm{MVDR}}^{H} R_{\mathbf{d d}} \mathbf{h h}^{H} R_{\mathbf{d d}}^{*} \mathbf{w}_{\mathrm{MVDR}}}{\mathbf{w}_{\mathrm{MVDR}}^{H} \mathbf{R}_{\mathbf{x x}} \mathbf{w}_{\mathrm{MVDR}}},
$$

where $|\cdot|$ is the absolute value. The underlying Gaussian variance is $\sigma_{T_{\mathrm{MVDR}}}^{2}=\frac{1}{2 K}$. Then, the closed-form solutions for both false alarm and detection probabilities are

$$
P_{\mathrm{d}}(\gamma)=\mathrm{Q}_{1}\left(\frac{\sqrt{\delta_{T_{\mathrm{MVDR}} ; \mathcal{H}_{1}}}}{\sigma_{T_{\mathrm{MVDR}}}}, \frac{\sqrt{\gamma}}{\sigma_{T_{\mathrm{MVDR}}}}\right)
$$

and

$$
P_{\mathrm{fa}}(\gamma)=\exp \left\{\frac{-\gamma}{2 \sigma_{T_{\mathrm{MVDR}}}^{2}}\right\}
$$

where $\mathrm{Q}_{1}$ is the generalized Marcum Q-function of order 1.

It is possible to particularize $\delta_{T_{\mathrm{MVDR}} ; \mathcal{H}_{1}}$ inserting (17) in (22). After a straightforward mathematical manipulation, the following expression is obtained:

$$
\delta_{T_{\mathrm{MVDR}} ; \mathcal{H}_{1}}=\frac{\left(\mathbf{h}_{0}^{H} \mathbf{R}_{\mathbf{x x}}^{-1} \mathbf{h}\right)^{2}}{\mathbf{h}_{0}^{H} \mathbf{R}_{\mathbf{x x}}^{-1} \mathbf{h}_{0}}
$$

where we assume that $\hat{\mathbf{R}}_{\mathbf{x x}} \simeq \mathbf{R}_{\mathbf{x x}}$, which is realistic in GNSS since the sample covariance matrix can be computed using a large number of snapshots ${ }^{\mathrm{b}}$. Notice that if the signal DOA is perfectly known $\left(\mathbf{h}_{0}=\mathbf{h}\right)$, then $\delta_{T_{\mathrm{MVDR}}}=\mathbf{h}^{H} \mathbf{R}_{\mathbf{x x}}^{-1} \mathbf{h}^{H}$; thus, $\delta_{T_{\mathrm{MVDR}}}=\delta_{T_{\mathrm{GL}}}$. However, in this particular case, $T_{\mathrm{MVDR}}$ outperforms $T_{\mathrm{GL}}$ since the $\chi^{2}$ degrees of freedom is fixed to 2. The theoretical PDF for the MVDR test statistic was validated by MC simulations, as shown in Figure 4.

\subsection{Blind null-steering beamformer}

As highlighted in Section 1, in the acquisition stage, it is difficult to provide the signal DOA information (which depends on the array location and attitude) required by the MVDR beamformer. However, it is possible to filter out the interferences using a blind null-steering beamformer (see, e.g., [15]). This approach presupposes that the GNSS signals are well below the noise floor, and thus, their contribution to the array covariance matrix is negligible. The algorithm minimizes the beamformer output power by nulling the directions where interfering signals are present. This beamformer is a particular case of the MVDR beamformer, where the steering vector is defined as $\mathbf{h}_{0}=\mathbf{h}_{\text {ref }}=\left[\begin{array}{llll}1 & 0 & \ldots & 0\end{array}\right]^{T} \in \mathbb{C}^{N \times 1}$. 


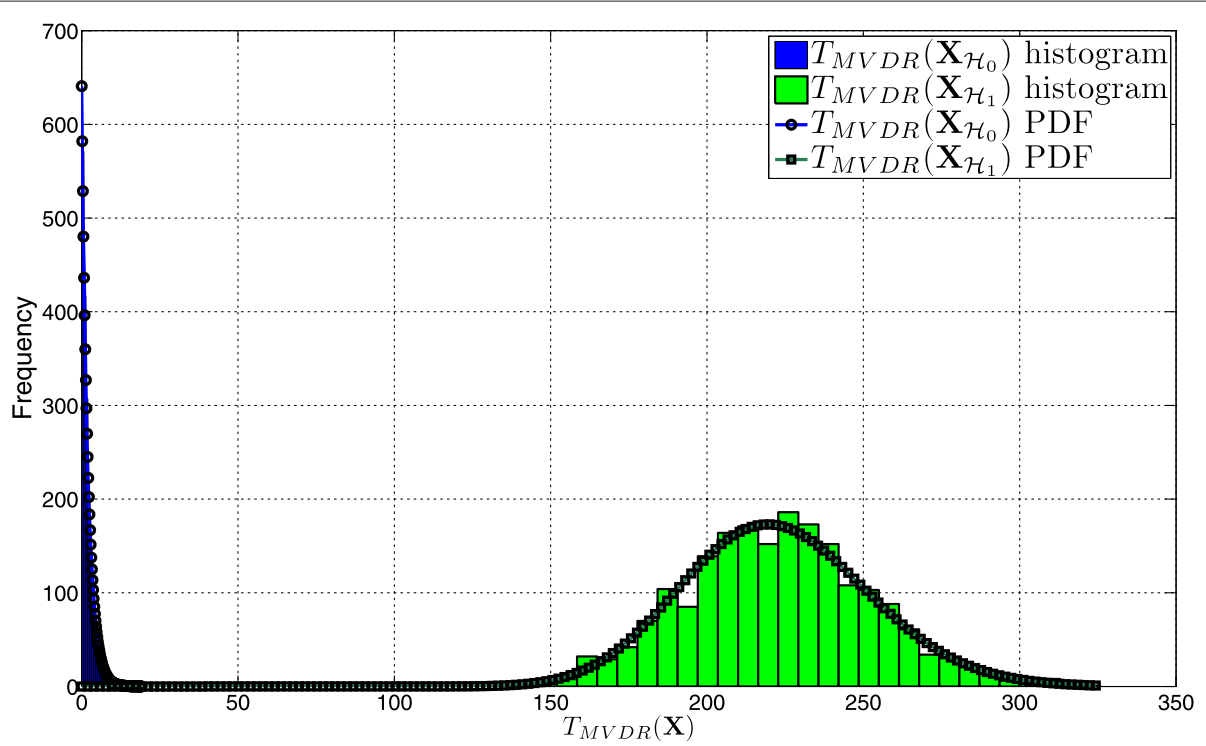

Figure $4 T_{\mathrm{MVDR}}(\mathrm{X})$ test statistic histograms and theoretical PDFs for 4,000 realizations and $\mathrm{CN} 0=34 \mathrm{~dB}-\mathrm{Hz}$ without interferences.

The behavior of the detector in the absence of interferences can be obtained by setting $\mathbf{P}_{\mathbf{H}_{\mathrm{i}}}^{\perp}=\mathbf{I}$ in (18) and assuming $\left|R_{\mathbf{d d}}\right|^{2} \simeq 1$ as follows:

$$
T_{\mathrm{NULL}}(\mathbf{y})=\frac{\sigma^{-2}}{\mathbf{h}_{\mathrm{ref}}^{H} \mathbf{h}_{\mathrm{ref}}}\left\|\hat{\mathbf{r}}_{\mathbf{x d}}^{H} \mathbf{h}_{\mathrm{ref}}\right\|^{2}=\frac{\left|\frac{1}{K} \mathbf{x}_{1} \mathbf{d}^{H}\right|^{2}}{\sigma^{2}}
$$

where $\mathbf{x}_{i}$ is the received signal vector for the $i$ th antenna element. In this particular case, the beamformer only activates the reference antenna and the resulting acquisition does not benefit from the available array gain.

Using the same procedure as in Section 4.2, the performance of the null-steering version of MVDR can be found inserting $\mathbf{h}_{0}=\mathbf{h}_{\mathrm{ref}}=\left[\begin{array}{lll}1 & 0 & \ldots\end{array}\right]^{T}$ in (25), obtaining

$$
\delta_{T_{\mathrm{NULL}} ; \mathcal{H}_{1}}=\frac{\left|\mathbf{r}_{\mathbf{x x}, 1} \mathbf{h}\right|^{2}}{\sigma_{1}^{2}},
$$

where $\mathbf{r}_{\mathbf{x x}, 1}=\left[R_{\mathbf{x}_{1} \mathbf{x}_{1}}, \ldots, R_{\mathbf{x}_{1} \mathbf{x}_{\mathbf{N}}}\right]$ is the first row of the autocorrelation matrix and $\sigma_{1}^{2}=R_{\mathbf{x}_{1} \mathbf{x}_{1}}$ is the signal power received with the reference antenna. Figure 5 shows the MC simulation validation for the null-steering test statistic theoretical PDF.

\section{Simulations}

The acquisition of a Galileo E1 signal was simulated for different carrier-to-noise density ratio (CNO) values in a scenario where a wideband, Gaussian noise-like in-band interference filtered to Nyquist frequency $\left(\frac{f_{s}}{2}\right)$ impinges into an array of $N=8$ elements, with uniformly distributed random DOA and interference-to-noise density ratio INO $=85 \mathrm{~dB}-\mathrm{Hz}$. The simulated array was circular with uniformly separated elements at $\frac{\lambda}{2}$ and without a central element. $\lambda$ was set to the GNSS L1/E1 carrier wavelength.

The baseband sampling frequency and the acquisition baseband bandwidth were set to 6 and $2 \mathrm{MHz}$, respectively. For each $\mathrm{CN} 0$ value, the simulation averages 10,000 independent realizations. The acquisition time was set to one PRN primary code period ( $T_{\text {acq }}=4 \mathrm{~ms}, K=24,000$ snapshots), and the $P_{\mathrm{fa}}$ was set to 0.001 for all the algorithms in order to set the particular threshold values. In addition, we considered different pointing errors in the DOA estimation for the MVDR beamformer in order to simulate a moderately uncalibrated array. The error in DOA estimation was modeled as a Gaussian additive error term with different mean values $\left(\mu_{e}=10^{\circ}, \mu_{e}=15^{\circ}\right.$, and $\mu_{e}=20^{\circ}$ ) in both azimuth and elevation angles, and $\sigma_{e}^{2}=5^{\circ}$ of variance in all the cases.

Figure 6 shows the acquisition performance in terms of $P_{\mathrm{d}}$. From the results, it can be inferred that $T_{\mathrm{GL}}(\mathbf{X})$ enjoys a higher performance than the power minimization beamformer algorithm $T_{\mathrm{NULL}}(\mathbf{y})$ due to the array gain, which is aligned with the theoretical performance expressions. If DOA information is available, $T_{\mathrm{MVDR}}(\mathbf{y})$ outperforms $T_{\mathrm{GL}}(\mathbf{X})$. Results show as well that the performance of $T_{\mathrm{MVDR}}(\mathbf{y})$ is severely affected by pointing errors.

The theoretical performance curves were plotted for all the analyzed algorithms using the analytical CDF expressions presented in Sections 3 and 4. We choose an ideal situation with no interference present in the scenario $\left(\mathbf{R}_{\mathbf{x x}}=\sigma^{2} \mathbf{I}\right)$, which are the algorithm performance upper bounds. Comparing the theoretical performance in this ideal scenario with the simulations, a performance reduction caused by the effect of the interference can be appreciated. 


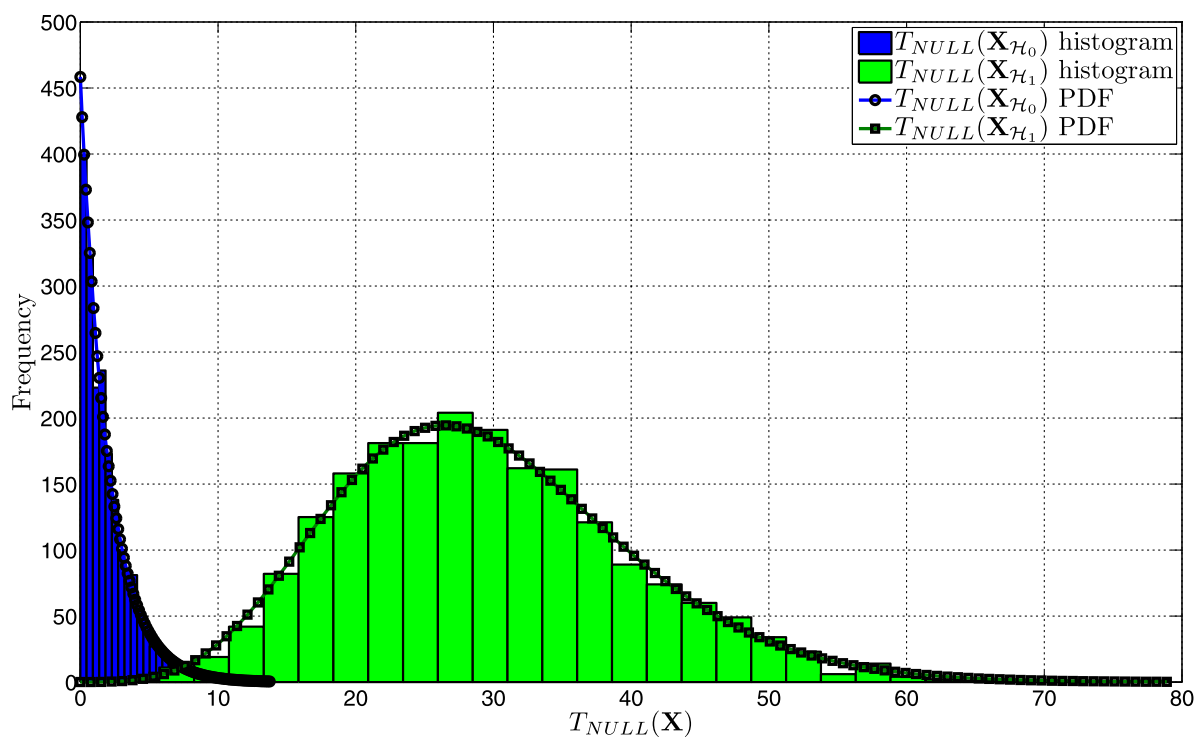

Figure $5 T_{\mathrm{NULL}}(\mathrm{X})$ test statistic histograms and theoretical PDFs for 4,000 realizations and CNO $=34 \mathrm{~dB}-\mathrm{Hz}$ without interferences.

\section{Computational complexity of the algorithms}

The proposed algorithms could be implemented in embedded digital processors that are mostly sharing resources with the rest of the GNSS receiver operations, specially in software-defined implementations. For that reason, it is important to analyze the computational complexity associated to each approach, as a complement of the performance analysis.

We consider the so-called asymptotic time complexity in the analysis. The time complexity of an algorithm can be viewed in terms of the number of basic operations it performs. For instance, we considered that a multiplication of two matrices of size $n_{1}$ by $n_{2}$ and $n_{2}$ by $n_{3}$ has an asymptotic operation cost of $\mathcal{O}\left(n_{1} n_{2} n_{3}\right)$. The meaning of this notation is that a function $f(n)$ is $\mathcal{O}(g(n))$ if and only if there exists a real, positive constant $C$ and a positive integer $n_{0}$ such that $f(n) \leq C g(n), \forall n \geq n_{0}$.

Table 1 shows the complexity associated to the basic operations involved in the proposed acquisition algorithms, such as the estimation of cross-correlation vectors, sample covariance matrix, and matrix inversion. The complexity analyses of the different test statistics are shown in Table 2. It can be seen that for a small number of antennas, compared to the number of snapshots $(K \gg N)$, the most demanding operation is the computation of both the spatial autocorrelation matrix $\hat{\mathbf{R}}_{\mathbf{x x}}$ and the cross-correlation vector $\hat{\mathbf{r}}_{\mathbf{x d}}$. In addition, we have taken into account the time delay and Doppler grid search

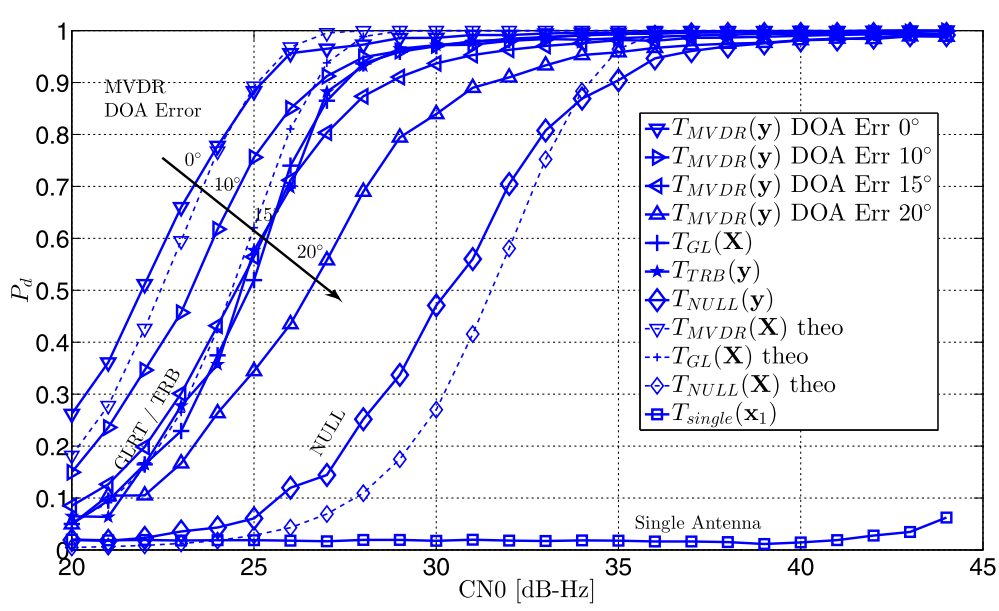

Figure 6 Acquisition performance in terms of $\boldsymbol{P}_{\mathbf{d}} \cdot P_{\mathrm{d}}$ Vs. CNO for Galileo E1B signal acquisition in the presence of a wideband interference with $\mathrm{INO}=85 \mathrm{~dB}-\mathrm{Hz}$ for different algorithms and DOA pointing errors. 
Table 1 Computational complexity of basic operations

\begin{tabular}{lccc}
\hline Computation & Operation & Size & Cost \\
\hline$\hat{\mathbf{R}}_{\mathbf{x x}}=\frac{1}{K} \mathbf{X \mathbf { X } ^ { H }}$ & Matrix-matrix product & $N \times K \times K \times N$ & $\mathcal{O}\left(N^{2} K\right)$ \\
$\hat{\mathbf{r}}_{\mathbf{x d}}=\frac{1}{K} \mathbf{X} \mathbf{d}^{H}$ & Matrix-vector product & $N \times K \times K \times 1$ & $\mathcal{O}(N K)$ \\
$\hat{\mathbf{R}}_{\mathbf{x x}}^{-1}$ & Matrix inversion & $N \times N$ & $\mathcal{O}\left(N^{3}\right)$ \\
\hline
\end{tabular}

operation. The total number of grid cells is represented as $L$, which depends on the grid search strategies (see, e.g., [16]). For instance, considering that the time delay grid granularity should be set to half-chip and the Doppler grid can be set to $250 \mathrm{~Hz}$, with a search margin equal to -5 to $+5 \mathrm{kHz}$, it gives $L \geq 40 \mathrm{~K}$.

Considering only the most significant terms, the complexity of the GLRT, TRB, and MVDR can be written as $\mathcal{O}_{\mathrm{GLRT}}\left(\left(N K+N^{2}\right) L+N^{2} K+N^{3}\right), \mathcal{O}_{\mathrm{TRB}}\left(2\left(N K+N^{2}\right) L+\right.$ $\left.N^{2} K+N^{3}\right)$, and $\mathcal{O}_{\mathrm{MVDR}}\left(K L+N^{2} K+N^{3}\right)$, respectively. The implementation of the TRB acquisition algorithm requires extra correlations due to the beamformer structure and, thus, higher computational cost than the GLRT. The analysis shows that the TRB algorithm doubles the computational complexity of the GLRT algorithm; however, both algorithms perform equally.

On the other hand, the MVDR-based test function only requires the computation of $\hat{\mathbf{R}}_{\mathbf{x x}}$ and the correlation of

Table 2 Computational complexity of acquisition algorithms

\begin{tabular}{lcrc}
\hline Algorithm & Test statistic & Operation & Cost \\
\hline GLRT & $\hat{\mathbf{r}}_{\mathbf{x d}}$ & Matrix $\times$ Vector & $\mathcal{O}(N K L)$ \\
$\hat{\mathbf{R}}_{\mathbf{x x}}$ & Matrix $\times$ Matrix & $\mathcal{O}\left(N^{2} K\right)$ \\
$\hat{\mathbf{R}}_{\mathbf{x x}}^{-1}$ & Matrix inversion & $\mathcal{O}\left(N^{3}\right)$ \\
TRB & $T(\mathbf{X})=\hat{\mathbf{r}}_{\mathbf{x d}}^{H} \hat{R}_{\mathbf{d d}}^{-1} \hat{\mathbf{R}}_{\mathbf{x x}}^{-1} \hat{\mathbf{r}}_{\mathbf{x d}}$ & Matrix-vector & $\mathcal{O}\left(N^{2} L+N L\right)$ \\
$\hat{\mathbf{r}}_{\mathbf{x d}}$ & Matrix $\times$ Vector & $\mathcal{O}(N K L)$ \\
$\hat{\mathbf{R}}_{\mathbf{x x}}$ & Matrix $\times$ Matrix & $\mathcal{O}\left(N^{2} K\right)$ \\
$\hat{\mathbf{R}}_{\mathbf{x x}}^{-1}$ & Matrix inversion & $\mathcal{O}\left(N^{3}\right)$ \\
$\hat{\mathbf{w}}=\hat{\mathbf{R}}_{\mathbf{x x}}^{-1} \hat{\mathbf{r}}_{\mathbf{x d}}$ & Matrix $\times$ Vector & $\mathcal{O}\left(N^{2} L\right)$ \\
$\mathbf{y}=\hat{\mathbf{w}}^{H} \mathbf{X}$ & Vector $\times$ Matrix & $\mathcal{O}(N K L)$ \\
$R_{\mathbf{y d}}=\frac{1}{K} \mathbf{y} \mathbf{d}^{H}$ & Vector $\times$ Vector & $\mathcal{O}(K L)$ \\
$R_{\mathbf{y y}}=\mathbf{w}^{H} \hat{\mathbf{R}}_{\mathbf{x x}} \mathbf{w}$ & Vector $\times$ Matrix & $\mathcal{O}\left(N^{2} L+N L\right)$ \\
$T(\mathbf{y})=\frac{\hat{R}_{\mathbf{y d}}^{H} \hat{R}_{\mathbf{y d}}}{\hat{R}_{\mathbf{y y}}}$ & Scalar & $\mathcal{O}(2 L)$ \\
$\hat{\mathbf{R}}_{\mathbf{x x}}$ & Matrix $\times$ Matrix & $\mathcal{O}\left(N^{2} K\right)$ \\
$\hat{\mathbf{R}}_{\mathbf{x x}}^{-1}$ & Matrix inversion & $\mathcal{O}\left(N^{3}\right)$ \\
$\hat{\mathbf{w}}=\frac{\hat{\mathbf{R}}_{\mathbf{x}}^{-1} \mathbf{h}_{0}}{\mathbf{h}_{0}^{H} \hat{\mathbf{R}}_{\mathbf{x x}}^{-1} \mathbf{h}_{0}}$ & Matrix $\times$ Vector & $\mathcal{O}\left(2 N^{2}+3 N\right)$ \\
$\mathbf{y}=\hat{\mathbf{w}}^{H} \mathbf{X}$ & Vector $\times$ Matrix & $\mathcal{O}(N K)$ \\
$R_{\mathbf{y d}}=\frac{1}{K} \mathbf{y d} \mathbf{d}^{H}$ & Vector $\times$ Vector & $\mathcal{O}(K L)$ \\
$R_{\mathbf{y y}}=\mathbf{w}^{H} \hat{\mathbf{R}}_{\mathbf{x x}} \mathbf{w}$ & Vector $\times$ Matrix & $\mathcal{O}\left(N^{2}+N\right)$ \\
$T(\mathbf{y})=\frac{\hat{R}_{\mathbf{y d}}^{H} \hat{R}_{\mathbf{y d}}}{\hat{R}_{\mathbf{y y}}}$ & & Scalar & $\mathcal{O}(2 L)$ \\
\hline & & &
\end{tabular}

the beamformer output stream with a local satellite signal replica, thus reducing the complexity at expenses of requiring an estimation of the signal DOA. The blind nullsteering beamformer can be considered a particular case of the GLRT, and the complexity associated to the algorithm can be reduced taking into account the particular structure of the steering vector $\mathbf{h}_{0}=\mathbf{h}_{\text {ref }}=\left[\begin{array}{llll}1 & 0 \ldots 0\end{array}\right]^{T}$.

\section{Conclusions}

We addressed the GNSS signal acquisition problem using antenna arrays for interference mitigation with two different approaches. The first approach operates directly on the array signal samples, performing a statistical detection of the satellite signal using a GLRT-based test function. The second one relies on a spatial filter that rejects the interferences before applying a conventional single-antenna acquisition algorithm. Different beamweight designs, including TRB, MVDR, and blind null-steering solutions, were analyzed in terms of detection and false alarm probabilities, and we found that the single-antenna-based acquisition after the TRB algorithm is mathematically equivalent to the antenna array-based GLRT detection. Thus, we show that different array processing approaches, and thus different implementation options, yield the same performance. The computational complexity in terms of the number of operations was also analyzed, and the results show that the TRB algorithm implementation has higher computational costs than the GLRT algorithm. However, the TRB beamforming structure in acquisition provides some benefits since it can be reused by a tracking algorithm afterward.

In the simulations, the acquisition after the MVDR beamformer obtained the best performance in the presence of strong interferences when the satellite signal DOA is estimated with less than $15^{\circ}$ of error, for an eight-element circular array. In the presence of pointing errors (i.e., $\mu_{e} \geq 15^{\circ}$ ), the MVDR-based acquisition performance is dramatically degraded. On the other hand, the acquisition after the blind null-steering beamforming does not reach the performance offered by the MVDR, as predicted by the theoretical analysis. In contrast, the GLRT algorithm does not impose any DOA estimation requirements and offers protection against uncorrelated directional interferences, even if the array is moderately uncalibrated.

\section{Endnotes}

${ }^{a}$ For the sake of simplicity of the notation, we omit the maximization operation of the acquisition test function over the signal synchronization parameters $\left(f_{d}, \tau\right)$ and its dependency in the rest of the paper.

b At least one PRN code period is available $(K \geq 2,046$ for GPS L1 C/A and $K \geq 4,092$ for Galileo E1, using two samples per chip). 


\section{Competing interests}

The authors declare that they have no competing interests.

\section{Acknowledgements}

This work has been partially supported by the European Commission in the framework of the FP7 Network of Excellence in Wireless COMmunications NEWCOM\# (contract no. 318306) and by the Spanish Ministry of Economy and Competitiveness project SOSRAD (Ref. TEC2012-39143).

\section{Received: 21 December 2012 Accepted: 19 August 2013}

Published: 3 September 2013

\section{References}

1. The Royal Academy of Engineering, Global Navigation Space Systems: reliance and vulnerabilities. Tech. rep. (The Royal Academy of Engineering, London, 2011)

2. DP Shepard, TE Humphreys, AA Fansler, Evaluation of the vulnerability of phasor measurement units to GPS spoofing attacks. Int. J. Crit. Infrastructure Prot. 5(3-4), 146-153 (2012)

3. RH Mitch, RC Dougherty, ML Psiaki, SP Powell, BW O'Hanlon, JA Bhatti, TE Humphreys, Signal characteristics of civil GPS jammers, in Proceedings of the ION GNSS, Portland, 20-23 Sept 2011

4. Technical Working Group, LightSquared Technical Working Group final report. Tech. rep. (Federal Communications Commission, Washington, D.C., 2011)

5. J Arribas, C Fernández-Prades, P Closas, Antenna array based GNSS signal acquisition for interference mitigation. IEEE Trans. Aerosp. Electron. Syst. 49(1), 223-243 (2013)

6. European Space Agency/European GNSS Supervisory Authority, Galileo Open Service, Signal In Space Interface Control Document (OS SIS ICD). Tech. rep. [Galileo Joint Undertaking] (European Space Agency/European GNSS Supervisory Authority, Prague, 2010)

7. E Kaplan, C Hegarty, Understanding GPS. Principles and Applications, 2nd edn (Norwood, Artech House, 2005)

8. SM Kay, Fundamentals of Statistical Signal Processing: Detection Theory (Prentice-Hall, Upper Saddle River, 1998)

9. G Casella, RL Berger, Statistical Inference, 2nd edn (Duxbury Press, Pacific Grove, 2001)

10. JBY Tsui, Fundamentals of Global Positioning System Receivers. A Software Approach (Wiley, New York, 2000)

11. RJ Muirhead, Aspects of Multivariate Statistical Theory (Wiley, New York, 1982)

12. J Arribas, GNSS array-based acquisition: theory and implementation. PhD thesis, Universitat Politècnica de Catalunya (UPC), 2012

13. RA Monzingo, TW Miller, Introduction to Adaptive Arrays (Wiley, New York, 1980)

14. M Cuntz, LA Greda, M Heckler, A Konovaltsev, M Meurer, Architecture of a real-time safety of life receiver, in Proceedings of the ION GNSS 2009, Savannah, 22-25 Sept 2009

15. MD Zoltowski, AS Gecan, Advanced adaptive null steering concepts for GPS, in IEEE Military Communications Conference, 1995. MILCOM 1995, vol. 3 (IEEE, Piscataway, 1995), pp. 1214-1218

16. D Borio, A statistical theory for GNSS signal acquisition. PhD thesis, Politecnico Di Torino, 2008

\section{Submit your manuscript to a SpringerOpen ${ }^{\odot}$ journal and benefit from:}

- Convenient online submission

- Rigorous peer review

- Immediate publication on acceptance

- Open access: articles freely available online

- High visibility within the field

- Retaining the copyright to your article

Submit your next manuscript at $\boldsymbol{\nabla}$ springeropen.com 